\title{
Odontogenic Maxillary Sinusitis - Need for Multidisciplinary Approach- A Review
}

\author{
Dr. Kumaran Alias Ramesh Colbert, Ms, Dnb (ENT) ${ }^{1}$, Dr. Devakumari, \\ $\mathrm{MDS}^{2}$, Dr. Ravi Sankar, Ms (ENT) ${ }^{3}$ \\ ${ }^{1}$ (Associate Professor\& HOD, Department of Otorhinolaryngology, Indira Gandhi Medical College \& \\ Research Institute (Government of Puducherry Institution), PUDUCHERRY - 605 009, PONDICHERRY \\ UNIVERSITY, INDIA) \\ ${ }^{2}$ (Assistant Professor, Department of Dentistry, Indira Gandhi Medical College \& Research Institute \\ (Government of Puducherry Institution), PUDUCHERRY - 605 009, PONDICHERRY UNIVERSITY, \\ INDIA) \\ ${ }^{3}$ (Assistant Professor, Department Of Otorhinolaryngology,Sri Manakula Vinayagar Medical College And \\ Hospital ,Madagadipet, PUDUCHERRY - 605 107, PONDICHERRY UNIVERSITY, INDIA).
}

\begin{abstract}
Odontogenic maxillary sinusitis (OMS) is a very common disease.Most of the cases present or referred to otorhinolaryngologists by General physicians and from other specialties.Many otorhinolaryngologists fail to find the exact etiology especially in the early cases having dental origin.Failure to detect this leads to failure of medical and surgical treatment by otorhinolaryngologist. Opinion from Dental Surgeon before treatment helps to find or rule out odontogenic pathology as its helps to decide type of medical and surgical intervention.This article reviews the common clinical features and the management protocols which will help the otolaryngologists ,Dental Surgeons \& other Specialists to efficiently treat odontogenic Maxillary Sinusitis, thereby preventing recurrence, spread to other sinuses \& complications.

Keywords: Odontogenic infection, sinusitis, maxillary sinus.
\end{abstract}

\section{Introduction}

Odontogenic maxillary sinusitis is a very common disease encountered by otorhinolaryngologists accounting for about 10 to $12 \%$ of maxillary sinusitis(1).It can be acute or chronic.In both cases odontogenic origin should be looked for especially when the medical management fails and diagnostic nasal endoscopy does not reveal any obstructive pathology in the maxillary sinus ostia.

Common dental cause are dental abscess,periodontal diseases,post dental extraction,oroantral fistula,undetected foreign bodies in antrum ${ }^{(2)}$.Common organisms are mixed aerobic and anaerobic organisms. Hence its important that not only antibacterial but also appropriate dental and /or otorhinolaryngologist surgical intervention is required. Disscusion with General Physician or other Specialists if there is associated systemic diseases like diabetes mellitus, hypertension, immunosuppressive conditions etc. coexists results in effective treatment. Recently researchers shown interest in odontogenic infections particularly like periodontitis ( chronic low grade infection due to microbial biofilm) because along with sinus complications it is important risk factor in the morbidity and mortality of systemic conditions like cardiovascular disease, diabetes and premature birth ${ }^{(3)}$

\section{Anatomy Of Maxillary Sinus \& Its Dental Relations}

The paranasal sinuses are group of air containing spaces present on either sides in the skull.The largest sinus is maxillary sinus. Floor of the maxillary sinus is formed by alveolar process and palatine process of maxilla. (fig1)

The premolars,first and second molars indent the floor of maxillary sinus and hence infections and others pathologies of these teeth affect the sinuses and present as sinus disease.(Fig2) .The bony wall, separating maxillary sinus from teeth roots varies from full absence, to teeth roots are covered only by mucous membrane. Sinus floor is situated about 3 to $5 \mathrm{~mm}$ below the nasal cavity floor .Maxillary sinus drains via the ostia situated in the medial wall in the middle meatus.About 4 to $5 \%$ have accessory ostia.

\section{Etiology Of Maxillary Sinuitis}

Most of the sinusitis are Rhinogenic in origin due to viral, bacterial or fungal infections. They cause oedema of the nasal mucosa and obstruct the drainage of the sinuses. 
Other causes can be anatomical obstruction of sinus by Deviated nasal septum, Allergic rhinosinusitis,Post traumatic sinusitis, Mucociliary clearance disorder like kartageners, young syndrome, Dental infections (4, 5).,Post extraction of teeth especially upper first ,second molars \& first premolars, odontogenic cysts(fig 3),periimplantitis(fig 4) General immunosuppressive status like uncontrolled diabetes mellitus,Acquired immune suppressive disorder are common predisposing and aggravating factors

\section{Microbiology}

Viral ,Bacterial,Fungal infections are common pathological agents.Itzak et al(1) study on microbiology of acute sinusitis showed the predominant aerobic organisms were $\alpha$-hemolystreptococci,microaerophilic streptococci, and Staphylococcus aureus. The predominant anaerobes were anaerobic Gram-negative bacilli ,Peptostreptococcus, and Fusobacterium spp.

In chronic sinusitis ,aerobes were recovered in $11 \%$, anaerobes only in $39 \%$, and mixed aerobic \& anaerobic bacteria in $50 \%$. The predominant aerobes were $\alpha$-hemolytic streptococci, microaerophilic streptococci, and S. aureus.The predominant anaerobes were Gram-negative bacilli, Peptostreptococcus \& and Fusobacterium spp $\beta$-lactamase-producing bacteria(BLPB) were recovered from $50 \%$ patients with acute sinusitis and from $75 \%$ patients with chronic sinusitis.

Fungal infections like Aspergillosis and Mucormycosis are common in immunocompromised individuals, but can also occur in non- immunocompromised. Recent study by rega et al(6) most common bacteria isolated were viridians streptococci, prevotella, staphylococci and peptosteptococcus.

\section{Clinical Features Of Odontogenic Maxillary Sinusitis}

Patients usually presents to Otorhinolaryngologist as the infection is rhinogenic origin in $90 \%$ of them.About $10 \%$ present to Dental surgeon.Acute sinusitis presents as facial pain over the maxillary sinus area,nasal obstruction ,nasal discharge,dental pain,general constituitional symptoms like fever,malaise ,head ache etc. ${ }^{(7)}$

Signs of acute sinusitis are tenderness over canine fossa,congested odematous nasal mucosa, since the nasal \& systemic symptoms predominate dental etiology may be missed.

Chronic odontogenic maxillary sinusitis presents as chronic nasal obstruction unilateral or bilateral,purulent nasal discharge decreased smell or foul smelling nasal discharge,post nasal drip,specific dental pain,bleeding while brushing teeth etc. ${ }^{(8)}$

Signs of chronic maxillary sinusitis are on anterior rhinoscopy pus in middle meatus, chronic nasal mucosal congestion, posterior rhinoscopy will reveal pus in middle meatus \& post nasal drip.Dental signs can be periodontitis, alveolitis,loose tooth with dental abscess,osteomyelitis etc. Acute and chronic OMS if not dignosed and treated properly,patient can present with spread of infection to other sinuses,orbit \& intracranial complication.

\section{Investigations}

A. X RAY: Routinely Waters view for maxillary sinus is done. It shows partial or complete haziness or mucosal thickening. Specific thickening in the floor of maxillary sinus should raise the suspicion of OMS.It also reveals dental fillings and implants approximately.

B. Orthopantomogram (OPG): The Orthopantomogram(fig5), utilizes three separate rotational axes but the "old" method is based on a single rotational axis. By means of the three axes, it is possible to make orthopantomograms, that is, the entire tooth-bearing area of the maxilla and mandible is projected on the film orthoradially and perpendicularly.In cases of OMS this film useful to detect dental pathology, which takes priority before undertaking any sinus surgery by Otolaryngologists. ${ }^{(9)}$

Uses of OPG:

Periodontal bone loss and periapical involvement.

Detecting source of dental pain

Assessment for the correct placement of dental implants

Caries detection especially in the inter-dental region.

Mucosal thickening in the floor of the maxillary sinus adjacent to the tooth involved. 


\section{COMPUTED TOMOGRAPHY(CT SCAN)}

Inflammatory disease, which causes resorption of bone, is readily seen on dental CT scans and can be divided into periodontal and endodontal disease (10). Frequently, both periodontal and endodontal disease coexist.(fig 6,\&7) The former affects predominantly the bone along the side of the root, and the latter, the bone at the root apex. Adjacent maxillary sinus \& other sinuses,any complications to orbit , Intracranial complications can be visualized in CT scan.This should be the modality of choice in immune compromised patients and patients who present clinically with complication irrespective of their immune status.

\section{Diagnostic nasal Endoscopy(DNE)}

This is done with rigid nasal endoscope to visualize any obstructive ,infective pathology in the nasal cavity especially the osteomeatal complex area for sinuses drainage. This endoscopy will reveal any evidence or suspicion of fungal sinusitis or Malignancy.

\section{Complications Of Oms}

Complication can be Extracranial ,Intracranial and Dental. Extra Cranial includes facial cellulitis or abscess,orbital cellulitis or intraorbital ,abscess especially when infection spreads to ethmoid sinuses. Intrancranial infections are meningitis, brain abscess,cavernous sinus thrombosis.

Dental complications are periodontal abscess,oroantral fistula etc.

\section{Treatment Of Oms}

As the cause of maxillary sinusitis is odontogenic origin treatment includes medical and surgical.

A. Medical treatment. In acute OMS due to bacterial sinusitis medical treatment is instituted to cover the aerobic organisms as they predominate in acute phase. In chronic phase mixed organisms aerobic and anaerobic organisms dominate, hence antibiotics to cover both should be given. Control of systemic disorder like diabetes mellitus should be done in consultation with Physician.

B. Surgical treatment: In acute phase may include dental extraction,drainage of dental abscess , antral wash is required. In chronic OMS after endo or periodontic pathology is treated ${ }^{(11)}$. If sinusitis persists Functional endoscopic sinus surgery to widen the natural maxillary sinus ostium and clear diseased muscosa from other sinuses if they are also involved. Tissues collected during surgery should be subjected to histopathological examination to rule any invasive fungal infection.

\section{Discussion}

Maxillary sinusitis is a very common of common disease that requires treatment of the sinusitis as well as of the as the etiology.Though most of the acute \& chronic sinusitis is of rhinogenic origin,OMS is often missed hence persistence of sinusitis or failure of sinus surgery occurs.

\section{Conclussion}

1. In all cases of sinusitis, odontogenic cause should looked for, clinically and radiologically.

2. As otorhinolaryngologists do not have expertise in detecting early dental pathology clinically and radilogically its good to get their expert opinion.

3. In patients with immunocompromised status, opinion and treatment as Physicians advice should advocated.

4. Medical treatment should cover Gram positive organisms and chronic sinusitis should cover mixed organisms.

5. Surgical treatment should include dental and sinus surgery

6. All materials collected during surgery should be subjected to histopathology to rule out fungus and malignancy.

\section{References}

[1]. Itzhak Brook MD, MSc, The Laryngoscope, Volume 115, Issue 5, pages 823-825, May 2005.

[2]. Mehra P, Murad H, Maxillary sinus disease of odontogenic origin,Otolaryngolologic Clinics of North America,2004 Apr;37(2):34.

[3]. Moutsopoulos NM, Madianos PN: Low-grade inflammation in chronic infectious diseases, paradigm of periodontal infections. Ann N Y Acad Sci 2006; 1088:251-264

[4]. Durr DG, Desrosiers MY. Chronic Sinusitis: An elusive diagnosis. Can J Diagnosis. 1998;15(12):75-81.

[5]. Abrahams JJ, Glassberg RM. Dental disease: Frequently unrecognized cause of maxillary sinus abnormalities. AJR 1996;166:12191223

[6]. Rega AJ, Aziz SR, Ziccardi VB, et al: Microbiology and antibiotic sensitivities of head and neck space infections of odontogenic origin. J Oral Maxillofac Surg 2006; 64:1377-1380

[7]. Maloney PL, Doku HC. Maxillary sinusitis of odontogenic origin. J Can Dent Assoc 1968; 34(11):591-603

[8]. Liebgott B. Dental considerations of the maxillary sinus. University of Toronto Dental Journal. 1(2):35-38,1998

[9]. Ransom JS. Sinusitis: Diagnosis and treatment. Medscape Respiratory Care 1997; 1(8) www.medscape.com 
[10]. James J. Abrahams, MD,Dental CT Imaging: A Look at the Jaw,a Review,Radiological society of north America,February 2010

[11]. Fabio Costa, MD, Endoscopic Surgical Treatment of Chronic Maxillary Sinusitis of Dental Origin,Journal of Oral and Maxillofacial SurgeryVolume 65, Issue 2, February 2007, Pages 223-228.

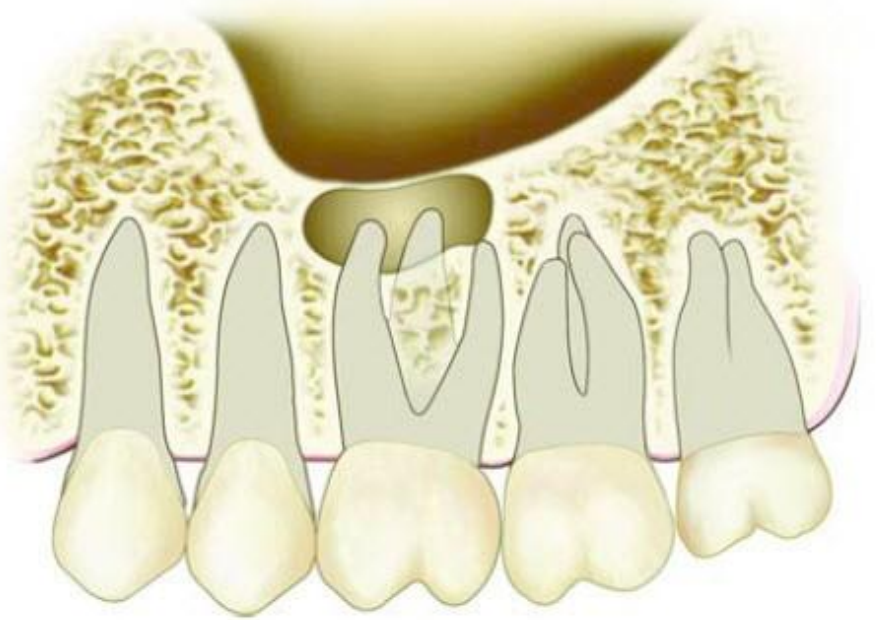

Figure 1: Relation of roots of molars \& premolar to maxillary sinus

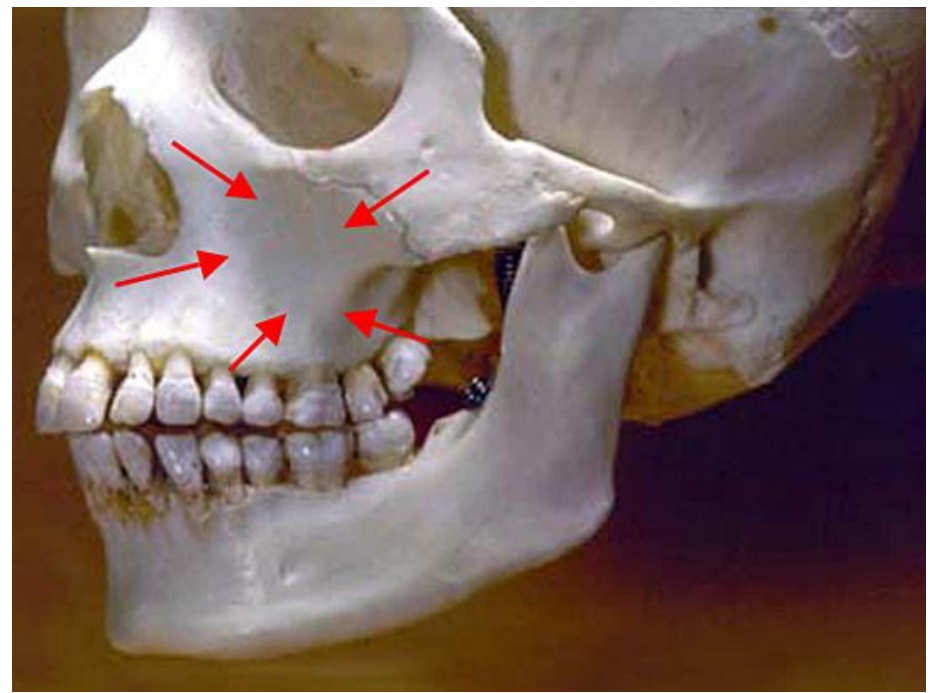

Figure 2: Relationship of molars and premolars to Maxillary sinus

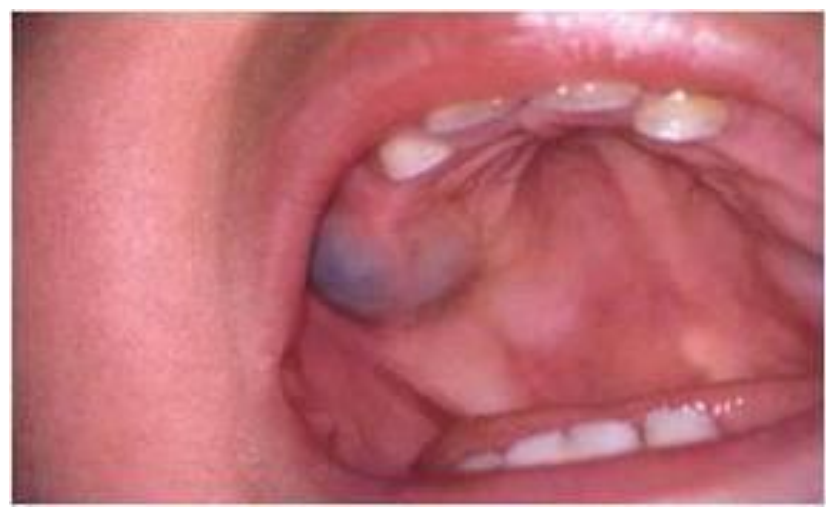

Figure 3: Odontogenic cyst 


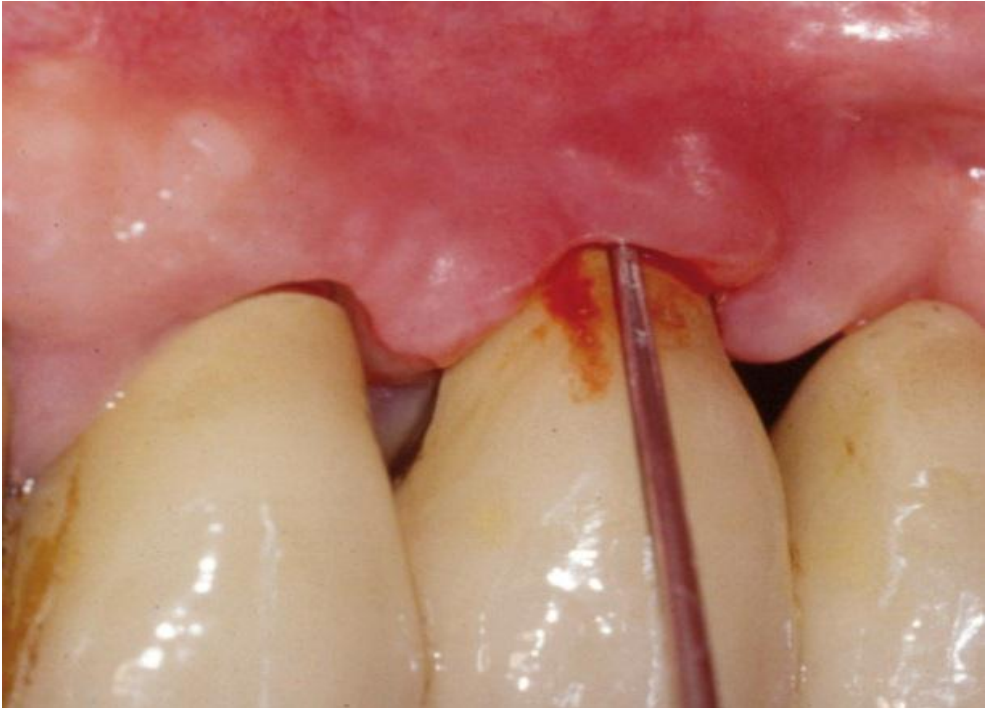

Figure 4: Periimplantitis

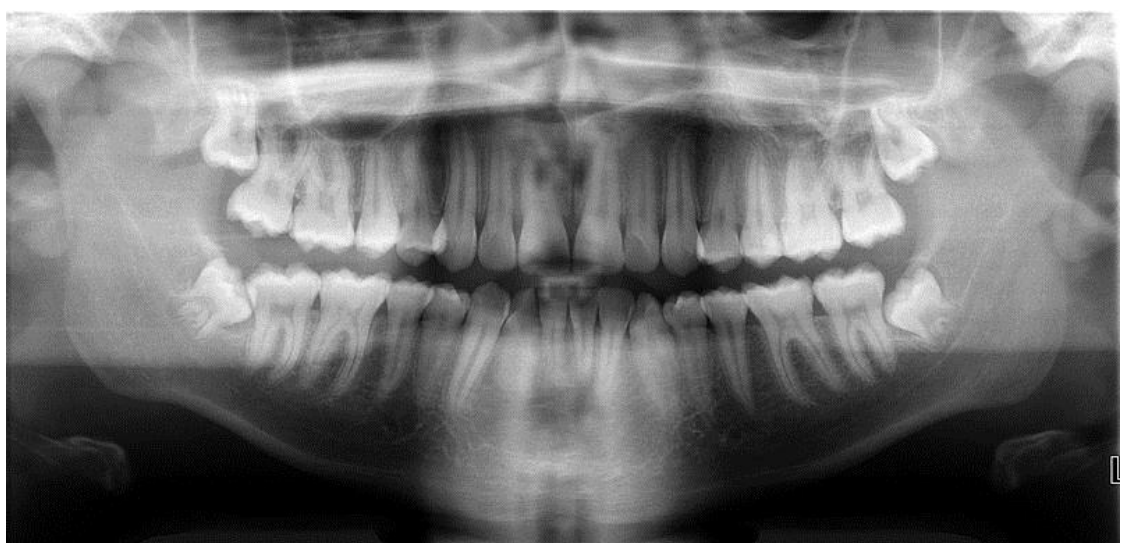

Figure 5: Normal orthopantomogram

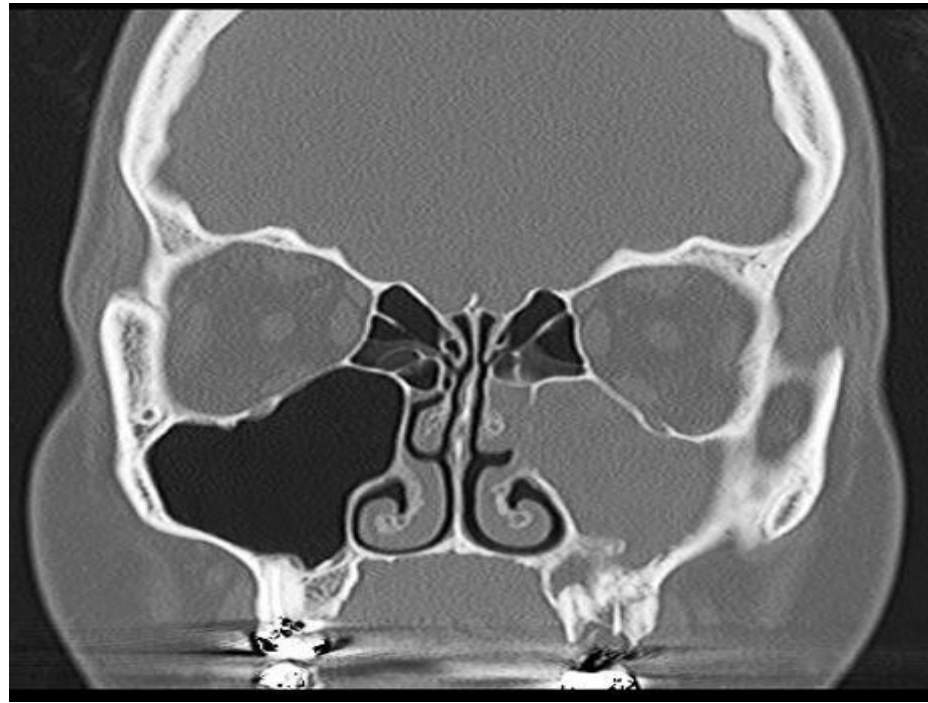

Figure 6: Left Periapical abscess with erosion of floor of Maxillary sinusis and complete opacity in maxillary sinus. 


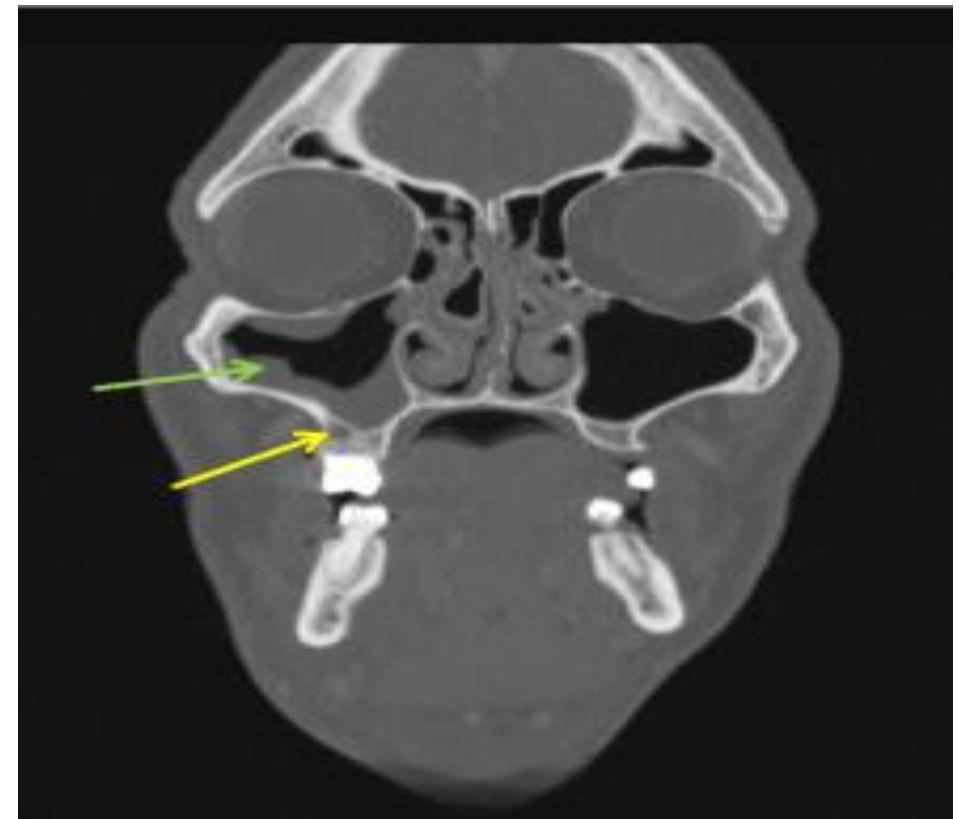

Figure 7: Green arrow mucosal thickening on the lateral \& floor of Right Maxillary Sinus. Yellow arrow shows periapical abscess. 